\title{
GSM based Water Management in Irrigation System Using ARM7
}

\author{
N.D. Pergad ${ }^{1}$, Y.P. Patil ${ }^{2}$ \\ ${ }^{1}$ Assistant Professor, Dept of Electronics and Tlecommunication Shree Tuljabhavani College of Engineering, Tuljapur, Osmanabad , India \\ ${ }^{2}$ Department of Electronics and Telecommunication Shree Tuljabhavani College of Engineering, Tuljapur, Osmanabad , India
}

\begin{abstract}
In India, agriculture plays an important role for development in food production. In our country, agriculture are depends on the monsoons which is not sufficient source of water. So the irrigation is used in agriculture field. In Irrigation system, depending upon the soil type, water is provided to plant. In this paper, automatic irrigation system based on ARMs and GSM module. The developed system is a machine based system, which automates the irrigation of land by combining various software and hardware approaches together to find exact field information and to provide instant across the field. This involves some sensors, LCD display, GSM and ARM7LPC2148 processor. LCD will be on field display purpose. GSM module will contains a Subscriber Identity Module (SIM) user can communicate with this SIM-Number. When the particular command activated or given by the user, immediately the corresponding sensor will activates and reads the present reading and immediately sends results to the same user mobile and displays in the LCD panel in the field. Immediately user will take the necessary action if required. Here we are using sensors to monitor the field condition. Those are Temperature, Level sensors. All these devices are connected to the ARM processor.GSM is used for communication purpose, with the help of AT (attention)- Commands we can communicate with the components. For level sensing applications we are using motor.
\end{abstract}

Keywords: Irrigation System, Soil Moisture, Sensor, Temperature Sensor, GSM

\section{Introduction}

Modern agriculture offers a range of benefits, including greater production and higher incomes for farmers including small producers in both developed and developing countries. Agriculture is a source of livelihood of majority Indians and has great impact on the economy of the country. In dry areas or in case of inadequate rainfall, irrigation becomes difficult. So, it needs to be automated for proper yield and handled remotely for farmer safety. Increasing energy costs and decreasing water supplies point out the need for better water management. Irrigation water management involves more than just turning on the irrigation system. Irrigation management is a complex decision-making process to determine when and how much water to apply to a growing crop to meet specific management objectives. If the farmer is far from the agricultural land he will not be noticed of current conditions. To overcome all the above disadvantages we are developing our system which is based on ARM7LPC2148 and GSM.

This work implements the emerging applications of GSM technology. Figure-1 shows the layout of a generic GSM network. Using GSM networks, a control system has been proposed that will act as an embedded system which can monitor and control an agricultural motor and other devices locally using built-in input and output peripherals. Controller and using microclimate data to schedule irri-gation water also irrigation is real time application. These technique, irrigate using following technique.

1) Internet based Monitoring using Servers, GPRS modems, etc. with different approaches.

2) GSM-SMS protocols using GSM module individually or in combination with Internet Technologies.

3) Monitoring using Wireless Sensor Networks.

4) Wireless Monitoring using Bluetooth, Wi-Fi, Zigbee and GSM.
5) Applications have varied widely like Home Automation, Security Systems, Bio-medical applications, Agriculture, En-vironment, Reservoir, Bridge health monitoring, etc.

\section{Literature Review}

In this paper, soil moisture sensor, temperature sensors placed in root zone of plant and gateway unit handles the sensor information and transmit data to a web application. One algorithm was developed for measure threshold values of temperature sensor and soil moisture sensor that was programmed into a microcontroller to control water quantity. For power photovoltaic panel was used. Another facto like cellular-Internet interface used that allowed for data inspection and irrigation scheduling to be programmed through a web page.

The automatic system was tested for 136 days and save 90 In this paper, soil moisture content has been detected using acoustic based technique was developed. The main propose of this technique is development for measure soil moisture in real time method. The technique based on relationship between two quantities i.e. speed of sound and the degree of saturation with water in soils. This experiment found that the speed of sound decreases with the moisture content following, depending on the kind of soil. [2]

This paper design a model of automatic irrigation system which is based on microcontroller and solar power was used only for source of power supply. Various sensor are placed in paddy field. Sensors sense water level continuously and give the information to farmer through cellular phone. Farmer controls the motor using cellular phone without going in paddy field. If the water level reaches at danger level, automatically motor will be off without conformation of farmer. [4]

The automatic system based on ARM and for communi-

\section{Volume 4 Issue 12, December 2015}




\section{International Journal of Science and Research (IJSR) \\ ISSN (Online): 2319-7064 \\ Index Copernicus Value (2013): 6.14 | Impact Factor (2014): 5.611}

cation GSM technology was used. Irrigation system provides foe adequate irrigation in particular area which is real time. Soil moisture sensor placed in root zone in paddy field and sense water level. The system was set up using ARM7TDMI core and GSM. GSM is an important part of these this system. System communicates using GSM. GSM operate through SMS and is a link between ARM processor and centralized unit. This system detects climate condition and field condition in real time. This information send to user in the form of SMS and GSM modem is controlled with the help of standard set of AT (Attention) commands. These commands are used to control majority of the functions of GSM model. [5]

In the paper, automatic irrigation technique irrigated using wireless sensor network i.e. Zig-bee and internet technology. The idea was developed for improve irrigation system and reduced cost of irrigation water. Sensors are placed in farm and sense continuously and collect he information. This information stored at center monitor and also passes to data collection interface and then transmits to the wireless sensor node. Using this information system was control automatically using internet. [6]

\section{Overall Analysis}

An automatic irrigation system used for irrigate sage crop field for 136 days and save 90

For automatic irrigation systems irrigate using cellular phone and for power source used solar power [7]. Arm also used for monitoring the irrigation system in real time based and for irrigation system, system irrigates using GPRS system [8].

Automatic irrigation system control using Zigbee and internet thing[9]. GPRS technique has some disadvantage viz speed, distance factor, reliability, so GPRS is not used in our project. Zigbee also have disadvantage i.e. low transmission rate. It is only use for smaller distance. Maximum papers have problem in networking and also some security issues [10].

\section{Proposed Work}

The main objective of the project is provide Water Management In Irrigation System with monitor and control the parameters in agriculture sectors. However, a large number of devices dont have the network interface and the data from them cannot be transmitted in network. A design of ARM7 processor-based embedded GSM interface is presented.

\section{System Description}

The following figure- 1 shows the block diagram of modern agricultural system. The parameters as discussed in the block diagram will monitor the field and gives the accurate results to the user. Here the new and important things are Level sensors to monitor the soil condition. If it is dry means, this condition is very harmful to plants. So immediately release the water into the soil and make it wet. For this we will use the level sensors. Temperature and humidity are useful in the case of monitoring the weather conditions. The LPC2148 microcontrollers are based on a 32-bit ARM7TDMI with real-time emulation and embedded trace support that combine microcontroller with embedded high speed flash memory upto $512 \mathrm{kB}$. A 128 - bit wide memory interface and unique accelerator architecture enable 32-bit code execution at the maximum clock rate. Due to their tiny size and low power consumption, LPC2148 are ideal for applications where miniaturization is a key requirement, such as access control and point-of- sale. Serial communications interfaces ranging from a USB 2.0 Full-speed device, multiple UARTs, SPI, SSP to I2C-bus and on-chip SRAM of $8 \mathrm{kB}$ up to $40 \mathrm{kB}$, make these devices very well suited for communication gateways and protocol converters, soft modems, voice recognition and low end imaging, providing both large buffer size and high processing power. Various 32-bit timers, single or dual 10-bit ADC(s), 10-bit DAC, PWM channels and 45 fast GPIO lines with up to nine edge or level sensitive external interrupt pins make these microcontrollers suitable for industrial control and medical control.

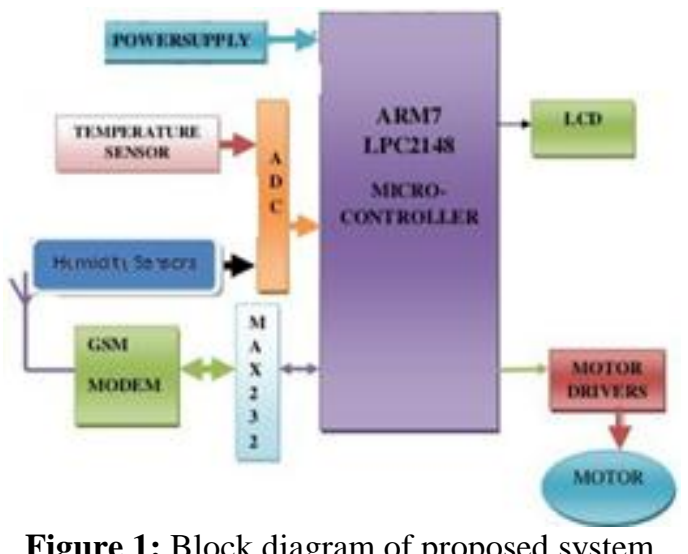

\section{A. Soil Moisture Sensor}

Measuring soil moisture is very important in agriculture to help farmer for managing the irrigation system. Soil moisture sensor is one who solves this. This sensor measures the content of water. Soil moisture sensor uses the capacitance to measure the water content of soil. It is easy to use this sensor. Simply insert this rugged sensor into the soil to be tested, and the volumetric water content of the soil is reported in percent.

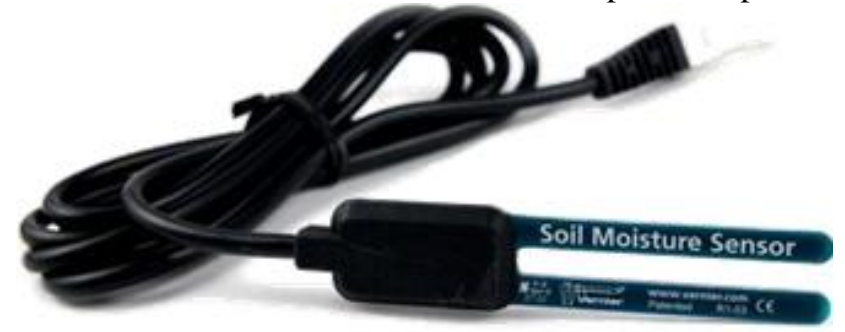

Figure 2: Soil Moisture Sensor

\section{B. LM35 Precision Centigrade Temperature Sensors}

In the temperature functional module we developed, we use the LM34 series of temperature sensors. The LM34 series are precision integrated-circuit temperature sensors, whose output voltage is linearly proportional to the Fahrenheit temperature. The LM34 thus has an advantage over linear temperature sensors calibrated in degrees Kelvin, as the user is not required to subtract a large constant voltage from its output to obtain convenient Fahrenheit scaling. The LM34 does not require any external calibration or trimming to

\section{Volume 4 Issue 12, December 2015}




\section{International Journal of Science and Research (IJSR) \\ ISSN (Online): 2319-7064 \\ Index Copernicus Value (2013): 6.14 | Impact Factor (2014): 5.611}

provide typical accuracies of $1.2 \mathrm{~F}$ at room temperature and $11.2 \mathrm{~F}$ over a full -50 to $+300 \mathrm{~F}$ temperature range. The LM34 is rated to operate over a -50 to $+300 \mathrm{~F}$ temperature range.

It is easy to include the LM34 series in a temperature measuring application. The output voltage of LM34 is linearly proportional to the Fahrenheit temperature, it has a Linear $+10.0 \mathrm{mV} / \mathrm{F}$ scale factor which means that you will get $\mathrm{n}^{*} 10.0 \mathrm{mV}$ output voltage if the environment temperature is $\mathrm{nF}$.

\section{Result}

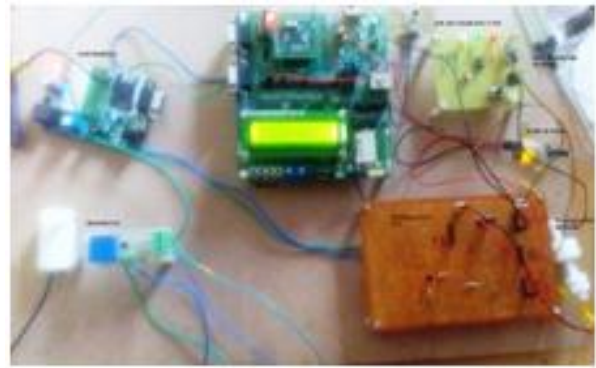

Figure 3: Transmitter Section

The results during observing, for testing the accuracy of the core system, we send an instruction as input to the ARM processor, through GSM using mobile, the result which is processed by the processor will be sent to the mobile and displayed on the LCD screen for different inputs from the sender.

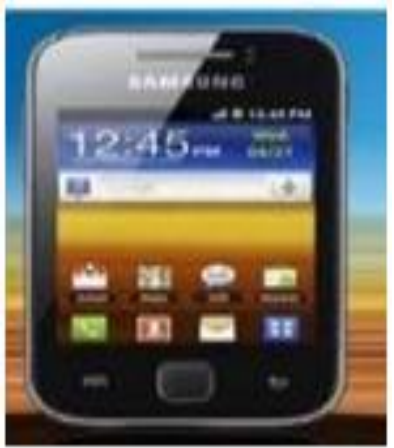

Figure 4: Receiver Section

\section{Conclusion}

The proposed model using ARM-LPC2148 which is fully based on GSM Module. This project developed agriculture field and increase the growth of food production and provides excellent water management . This can also useful for increas-ing the economy and demand of food necessity.

\section{References}

[1] Joaqun Gutirrez, Juan Francisco Villa-Medina, Alejandra Nieto-Garibay, and Miguel ngel Porta- Gndara Automated Irrigation System Using a Wireless Sensor Network and GPRS Module IEEE 2013.

[2] Thomas J. Jackson, Fellow, IEEE, Michael H. Cosh, Rajat Bindlish, Senior Member, IEEE, Patric J. Starks, David D. Bosch, Mark Seyfried, David C. Goodrich, Mary Susan Moran, Senior Member, IEEE, and Jinyang Du ,Validation of Advanced Microwave Scanning
Radiometer Soil Moisture Products, IEEE 2010.

[3] Jia Uddin, S.M. Taslim Reza, Qader Newaz, Jamal Uddin, Touhidul Islam, and Jong-Myon Kim,Automated Irrigation System Using Solar Power 2012 IEEE.

[4] R. Eaton, J. Katupitiya, K. W. Siew, and B. Howarth, Autonomous farming: Modeling and control of agricultural machinery in a unified framework, in Proc. 15th Int. Conf. Mechatronics and Machine Vision Practice, Auckland, New Zealand, Dec. 2008, vol. 1, pp. 499504.

[5] The ARM Microcontroller and Embedded Systems By Muhammad Ali Mazidi and Janice Gillispie Mazidi. Pearson Education.

[6] B. Astrand and A. Baerdveldt, A vision based rowfollowing system for agricultural field machinery, Mechatronics, vol. 15, no. 2, pp. 251269, 2005.

[7] Potts, J. and Sukittanon, S. (2012) Exploting bluetooth on android mobile mobile devices for home security application, proceedings of southeastcan, 15-18 March 2012, orlando, florida,USA.

[8] Kuniaki Umino, Yasuhiro Ohyama, Jin-Hua She and Hiroyuki Kobayashi, , Remote Controlled Embedded System, 4th China- Japan International Workshop on Internet Technology and Control Applications, Hunan, China, 21-26 October, 2005. 